\title{
Zum Begriff «Komplementärmedizin»
}

Auch als Mitglied der Schriftleitung habe ich nie verhehlt, dass ich dem Titel dieser Zeitschrift, nämlich dem Ausdruck «Komplementärmedizin», kritisch gegenüberstehe, ich selbst hätte ihn nicht gewählt. Komplementär sagt mir auf der einen Seite zu viel - teilweise auch etwas Falsches - auf der anderen Seite zu wenig.

Ich hätte mich - wie könnte ich anders - für «Naturheilkunde» entschieden, obwohl schon das «-kunde» manchen nach Ideologie, willkürlicher Interpretation und fraglichen Beweisen klingt. Dann hätten wir uns auf Naturheil-«verfahren» geeinigt, der Begriff ist unverfänglich, solange er mit Natur die Mittel und Methoden bezeichnet, mit welchen wir unsere Patienten behandeln: Erde, Wasser, Luft, Licht, Wärme, Bewegung, Nahrung, Heilpflanzen usw.

Denken wir aber an die «Natur» (die Physis) des Menschen selbst, ist diese Physis das Objekt einer Behandlung, betreiben wir also Physiotherapie im klassischen Sinne und mit den klassischen Konzepten, mag mancher kritische Mensch schon wieder den Kopf schütteln. Dann drücke ich mich modern aus, dann spreche ich von Habituation und Adaptation, von Training, Funktion und Rhythmus, «empowerment» des Patienten und den Prinzipien einer Salutogenese.

Bei der FORSCHENDEN KOMPLEMENTÄRMEDIZIN (z. B. in der Schriftleitung) fühle ich mich überwiegend immer noch zu Gast; im vierten Erscheinungsjahr dieser Zeitschrift kam das Angebot, sie als Publikationsorgan der neu zu gründenden Europäischen Gesellschaft für Klassische Naturheilkunde zu nutzen. Es bestand eine langjährige Bekanntschaft mit einem Teil der Schriftleitung, einige wurden bald auch in der Europäischen Gesellschaft aktiv. Vor kurzem dann der grosse Erfolg: Die Zeitschrift wurde in die wichtigen internationalen Registriersysteme aufgenommen. Man kann die Leistung der alten Garde um D. Melchart und K. Linde mit ihrer Chefredakteurin Marcela Ullmann gar nicht hoch genug einschätzen, wir haben uns alle sehr gefreut (s. Editorial der Schriftleitung in Heft 1, 1999).

Trotzdem erfahre ich von meinen naturheilkundigen Freunden immer wieder einmal Kritik, weil unsere wissenschaftlichen Themen in einer Zeitschrift mit einem umstrittenen Titel erscheinen. U. a. argumentiere ich, dass man über diesen Namen ja auch noch einmal nachdenken könnte, ich schlage vor: «Forschende Naturheil- kunde». Dann halten die Veteranen der Schriftleitung mir vor, dass ich, eingeladen und kaum an Bord des gut fahrenden Schiffes, dieses kapern und eine neue Fahne hissen will. Ich sehe ein, dass dieser Handstreich so nicht gelingen kann und sich so nicht gehört. Ich tröste mich: Vielleicht ist es ja nur noch zu früh. Ich sehe einen allgemeinen Trend in Richtung auf die klassischen und prizipiell verstehbaren Behandlungsmethoden.

Was habe ich gegen «komplementär»? Sehr einfach könnte man es mit «sich gegenseitig ergänzend» übersetzen, zu einem Ergänzen bedarf es aber mindestens zweier Akteure. Den einen Part, nämlich den, der ein Ergänzen zulässt, müsste ja wohl die sog. Schulmedizin spielen. Bei dieser ist Komplementärmedizin eine ungeliebte Braut, hier sollen wir uns und unserem Publikum nichts vormachen. Komplementär signalisiert einen Anspruch, der nicht zu halten ist, an dieser Stelle sagt mir der Ausdruck zu viel.

Tatsächlich hat komplementär in diesem Zusammenhang einen negativen Bedeutungswandel erfahren. Komplementär bezeichnet den Aussenseiter, ähnlich wie «alternativ» in der alternativen Medizin. Mit alternativ wird nicht ein zusätzliches, allgemein anerkanntes therapeutisches Angebot neben anderen Behandlungsmethoden bezeichnet, sondern ebenfalls die Tatsache des Aussenseiters. Niemand mag sein Verfahren so bezeichnen.

Vor allem leistet «komplementär» keinen Beitrag zu einer weiteren Differenzierung unter den vielen Methoden ausserhalb der medizinischen «Schule» - hier sagt mir der Ausdruck zu wenig. Er signalisiert ein Laisser-faire und eine Beliebigkeit, die niemandem nutzt. Extrem fragliche Methoden werden in der Gesellschaft anerkannter Verfahren aufgewertet, einigermassen plausible Konzepte werden in fragwürdiger Gesellschaft selbst fragwürdig.

Ähnliches geschieht jetzt in der offiziellen Gesundheitspolitik, hier werden die klassischen Naturheilverfahren zusammen mit den «komplementären» bzw. den «alternativen» Methoden unter «Unkonventionelle Medizinische Richtungen» (UMR) abgehandelt. Ich wehre mich dagegen [1].

Insofern ist es natürlich sehr wichtig und zu begrüssen, dass eine Zeitschrift sich mit dieser Medizin auch wissenschaftlich beschäftigt. Das ist ein anderer Aspekt, und insofern sollten meine natur-

\section{KARGER}

Fax +497614520714

E-mail kargergmbh@aol.com

www.karger.com (c) 1999 S. Karger GmbH, Freiburg

Accessible online at:

http://BioMedNet.com/karger 
heilkundigen Freunde bereit sein, sich und ihr Thema hier mit einzubringen. Leider ist das noch nicht sehr umfangreich der Fall.

Man könnte aber auch sehr viel höhere Inhalte und Ansprüche mit dem Terminus verbinden. 1927 hat Niels Bohr Komplementarität am Beispiel der gleichzeitigen Wellen- und korpuskulären Natur des Lichtes entwickelt, beide sind gültig und schliessen sich gleichzeitig gegenseitig aus: Im allgemeinen müssen wir darauf gefasst sein, so resümiert K. M. Meyer-Abich den Bohrschen Gedanken, «dass zur vollständigen Erfahrung eines Gegenstandes verschiedene Tat-Sachen, Gruppen von Tat-Sachen oder Weisen des Mitseins gehören und dass man von dieser Verschiedenheit - anders als in der klassischen Physik - nicht zu einer eindeutigen Beschreibung übergehen kann, in der vom jeweiligen Mitsein abgesehen wird» [2]. Meyer-Abich weist auf Parallelitäten zum Gestaltkreis Viktor v. Weizsäkkers: Hier besteht Komplementarität auch durch unterschiedliche Selbst- oder Selbstmiterfahrung. Diese Verhältnisse können übrigens auch für die Wahrnehmung und Bedeutungserteilung einzelner Naturheilmittel und Naturheilverfahren untersucht werden [3]. Mit den Ausführungen und Vorschlägen von Bohr und Weizsäcker werden Medizin und Naturheilkunde eine ungemein spannende Angelegenheit!

Es reizt mich, noch einen Blick auf das Polare oder die Polarität in der Natur zu werfen. Stark vereinfacht könnte man sie als das Gegenüber und das Verhältnis zwischen den unterschiedlichen Ausprägungen (den Polen) einer Qualität, einer Eigenschaft oder einer Kategorie bezeichnen. Bei Schelling ist es ein - unendlich sich abwandelnder - Streit entgegengesetzter Tendenzen, wo Heterogenes «sich anzieht» und Homogenes «sich zurückstösst». In diesem inneren Widerspruch sieht Schelling den Grund aller Tätigkeit der Natur, das Gesetz der Polarität ist das allgemeine Weltgesetz. Typische Polaritäten bilden bei Goethe das Licht und die Finsternis, das Innen und das Aussen, die Dauer und der Wechsel, das Ballen und das Lösen (Systole und Diastole) und schliesslich die zweierlei Gnaden, welche im Atemholen erlebt werden können.

Neben der Gleichzeitigkeit vorhandener Gegensätze zeigt er auf eine zeitliche Abfolge sich ablösender, gegensätzlicher Tendenzen: Er unterscheidet eine simultane und eine sukzessive Polarität. In dieser Polarität sieht Goethe das Wesen, die Bildung und Umbildung der Phänomene, in und aus dieser Polarität erkennt er das von ihm in die Naturwissenschaft eingeführte Prinzip der Steigerung. Polarität ist mehr dem Materiellen, Steigerung mehr dem
Geistigen angehörig. «Weil aber die Materie nie ohne Geist, der Geist nie ohne Materie existiert und wirksam sein kann, so vermag auch die Materie sich zu steigern, sowie sichs der Geist nicht nehmen lässt, anzuziehen und abzustossen» [4]. A. Schmidt sieht an dieser Stelle eine spezifisch deutsche Antwort auf die seit dem 17. Jahrhundert übliche statisch-geometrische Methode in der Naturwissenschaft.

Komplementarität und Polarität sind hervorragende Modelle, das medizinische Denken, die Wahrnehmung und Beurteilung von Kranken und Gesunden, aber auch von den Heilmitteln und -methoden der Natur lebendig zu erhalten, zu individualisieren und zu vergeistigen. Sie helfen zu einem Verständnis medizinischer Systeme, auch wenn diese gewöhnliche Naturwissenschaft überschreiten. Hier öffnet sich ein grosses Feld aufregender geisteswissenschaftlicher Betätigungen. Ich sehe Möglichkeiten, der «Natur» tatsächlich etwas näher zu kommen, manche moderne, pseudowissenschaftliche Erklärungsmodelle der alternativen Therapie empfinde ich ebenfalls als statisch-geometrisch und starr, auf keinen Fall aber als ganzheitlich oder «ganz» [5].

Bleiben wir bescheiden, noch einmal Goethe (zit. n. [4]): «Dass wir der Idee jenes Ganzen bedürfen, wenn wir die Welt erforschen, bedeutet nicht, dass wir seiner selbst je habhaft werden». Und an Windischman schrieb er am 28. Dezember 1812: «Sie bemerken mir recht, dass ich das Magische, das Höhere, Unergründliche, Unaussprechliche der Naturwirkungen zwar nicht mit Ungunst, aber doch von der negativen Seite betrachte; und so ist es auch».

Noch eine sehr frohe Botschaft am Schluss: Der Schriftleitung war es möglich, Herrn Prof. Klaus M. Meyer-Abich für eine Mitarbeit der Zeitschrift zu gewinnen. Ihm ein sehr herzliches Willkommen!

M. Bühring, Berlin

\section{Literatur}

1 Bühring M: Klassische Naturheilverfahren sind keine unkonventionelle Therapie. Münch Med Wochenschr 1998 a;140:570.

2 Meyer-Abich KM: Praktische Naturphilosophie. München, Ch. Beck, 1997.

3 Bühring M: Naturheilkunde: Grundlagen, Anwendungen, Ziele. München, Ch. Beck, 1997.

4 Schmidt A: Goethes herrlich leuchtende Natur. München, Carl Hanser, 1984.

5 Bühring M: Über Naturheilkunde und das «Ganze» in der Medizin. Dtsch Ärztebl 1998 b;95:343. 